\title{
E-Government in Sachsen-Anhalt - nur mit Datenschutz als Vertrauensfaktor
}

\section{Stellungnahme des Landesbeauftragten vor der Enquete-Kommission}

Der Landesbeauftragte für den Datenschutz und die Informationsfreiheit, Herr Dr. Harald von Bose, hatte am 19. April 2013 Gelegenheit, im Rahmen einer Anhörung der Enquete-Kommission des Landtages von Sachsen-Anhalt in öffentlicher Sitzung zu aktuellen Fragestellungen des Datenschutzes und der Informationssicherheit Stellung zu nehmen. Die Enquete-Kommission befasst sich unter dem Auftrag „Öffentliche Verwaltung konsequent voranbringen - bürgernah und zukunftsfähig gestalten“ mit der Zukunft der öffentlichen Verwaltung und behandelt u. a. Themen wie Funktionalreform, Personalstruktur und E-Government und Open Government.

Der Landesbeauftragte betonte dabei, dass Datenschutz stets Grundrechtsschutz sei, und wies insbesondere auf das vom Bundesverfassungsgericht entwickelte Grundrecht auf Gewährleistung der Vertraulichkeit und Integrität informationstechnischer Systeme hin. Für einen effektiven Grundrechts-schutz sind technische und rechtliche Aspekte zusammen zu betrachten.

In Sachsen-Anhalt hat es in den letzten 20 Jahren immer wieder strukturelle Veränderungen bei der Wahrnehmung der Aufgaben der IT-Koordinierung und des E-Government in der Landesverwaltung gegeben. Der Landesbeauftragte wurde beratend beteiligt, allerdings nicht stets vollständig und früh-zeitig. In den vergangenen Jahren wurden von der Landesregierung IT-Leitbilder, Strategien und Maßnahmenpläne entwickelt. Nach Einschätzung des Landesbeauftragten besteht der Eindruck, dass wichtige inhaltliche Fragen nur mit strukturellen Antworten versehen worden sind

In Sachsen-Anhalt erfolgte eine Konzentration der Zuständigkeiten für IKT-Strategie, E-Government und Dienstleistung, seit Jahren der geteilten Zuständigkeit in mehreren Ressorts, beim Ministerium der Finanzen. In diesem Zusammenhang erscheint es fraglich, ob die beabsichtigte Verlagerung aller Dienstleistungsaufgaben auf „Dataport“, einer Anstalt des öffentlichen Rechts, die mittlerweile für fünf Bundesländer tätig ist, alternativlos ist. Jedenfalls hätte es einer gründlicheren Analyse vor einer solchen Entscheidung bedurft.

Bei der Betrachtung der bisherigen IT-Strategie und der Strategie „Sachsen-Anhalt digital 2020“ fällt auf, dass viele der Vorhaben eher im innerstaatlichen Sektor angesiedelt sind, nicht aber den eigentlichen Kerngedanken eines modernen E-Government umsetzen, nämlich Projekte im Verhältnis Staat-Bürger.
Zusätzlich ist kritisch anzumerken, dass der Staat zu wenige vertrauensbildende Maßnahmen zugunsten des Grundrechtsschutzes ergreift. Mit der bereits im Jahr 2006 geschaffenen Sicherheitsinfrastruktur (PKI-LSA) wurden zwar die Voraussetzungen für eine flächendeckende Einführung und Nutzung der fortgeschrittenen und qualifizierten Signatur sowie für Verschlüsselungsaufgaben geschaffen. Der Landesbeauftragte gibt zu bedenken: „E-Government-Dienstleistungen werden von den Bürgerinnen und Bürgern nur genutzt, wenn sie Vertrauen in diese Dienste haben und darüber hinaus allgemein in die Datenverarbeitung des Staates.“ Der Landesbeauftragte empfiehlt, ein EGovernment-Gesetz für das Land zu schaffen.

Auch bei der Weiterentwicklung des informationstechnischen Netzes des Landes Sachsen-Anhalt (ITN-LSA) zum „ITN-XT“ kommt den Aspekten der Informationssicherheit und der Datensicherheit, insbesondere wenn es um sensible Steuer- und Sozialdaten geht, wesentliche Bedeutung zu. Der Landesbeauftragte fordert daher ein umfassendes Gesamtsicherheitskonzept für das zukünftige Landesnetz.

Eine moderne IKT-Strategie für das Land kann nur gelingen, wenn sie ganzheitlich, nachhaltig, verbindlich und vernetzt angelegt ist. Dazu gehört dringend die Einbindung der kommunalen Ebene.

E-Government ist auch entscheidende Voraussetzung für ein erfolgreiches Open Government. Dazu liegen seitens der Landesregierung bisher keine Strategieüberlegungen vor. Das Informationszugangsgesetz Sachsen-Anhalts gewährt einen voraussetzungslosen Informationszugangsanspruch zu allen amtlichen Informationen der öffentlichen Stellen. Die proaktive Veröffentlichungspflicht der Behörden sollte auch im Sinne von mehr Transparenz, E-Partizipation und Kooperation durch eine zentrale Online-Plattform des Landes ergänzt werden. Andere Länder sind hier weiter mit der Einrichtung von solchen Informationsregistern. Darauf hat der Landesbeauftragte auch bereits in seinem II. Tätigkeitsbericht zur Informationsfreiheit vom März 2013 hingewiesen.

Dr. Harald von Bose, Landesbeauftragter für den Datenschutz und die Informationsfreiheit Sachsen-Anhalt 